\title{
Load and Energy Aware Hybrid Routing Protocol for Hybrid Wireless Mesh Networks
}

DOI:

10.1109/VTCFall.2019.8891351

\section{Document Version}

Accepted author manuscript

Link to publication record in Manchester Research Explorer

\section{Citation for published version (APA):}

Chai, Y., \& Zeng, X. (2019). Load and Energy Aware Hybrid Routing Protocol for Hybrid Wireless Mesh Networks. In 2019 IEEE 90th Vehicular Technology Conference (VTC2019-Fall) (pp. 1-5)

https://doi.org/10.1109/VTCFall.2019.8891351

\section{Published in:}

2019 IEEE 90th Vehicular Technology Conference (VTC2019-Fall)

\section{Citing this paper}

Please note that where the full-text provided on Manchester Research Explorer is the Author Accepted Manuscript or Proof version this may differ from the final Published version. If citing, it is advised that you check and use the publisher's definitive version.

\section{General rights}

Copyright and moral rights for the publications made accessible in the Research Explorer are retained by the authors and/or other copyright owners and it is a condition of accessing publications that users recognise and abide by the legal requirements associated with these rights.

\section{Takedown policy}

If you believe that this document breaches copyright please refer to the University of Manchester's Takedown Procedures [http://man.ac.uk/04Y6Bo] or contact uml.scholarlycommunications@manchester.ac.uk providing relevant details, so we can investigate your claim.

\section{OPEN ACCESS}




\title{
Load and Energy Aware Hybrid Routing Protocol for Hybrid Wireless Mesh Networks
}

\author{
Yuan Chai and Xiao-Jun Zeng \\ School of Computer Science, the University of Manchester, Manchester, UK, M13 9PL \\ Email: yuan.chai-2@ postgrad.manchester.ac.uk, x.zeng@manchester.ac.uk
}

\begin{abstract}
Effective routing protocols considering network features can improve network performance dramatically. As hybrid wireless mesh network (WMN) is composed of static mesh routers and mobile mesh clients, hybrid routing protocol fits hybrid WMN well. However, the existing hybrid routing protocols designed for hybrid WMN do not fully consider the regional network condition, and as a result cannot effectively improve network performance as a whole. In this paper, a load and energy aware hybrid routing protocol (LE-HRP) is proposed. In LE-HRP, gateway- and clientoriented traffic are considered respectively. The queuing delay which can measure the load condition is taken into account in both traffic types. In the process of calculating delay, both physical and logical interferences are considered. In addition, for client-oriented traffic, as energy of mesh clients is limited, in addition to reducing delay, the energy efficiency is also improved in the meantime. A mesh client with low energy can still be used if it can help the network reduce the delay significantly and sufficiently. Otherwise, a mesh client with more residual energy will be chosen. Simulation results through NS3 show that LE-HRP achieves better network performance.
\end{abstract}

\section{INTRODUCTION}

Hybrid wireless mesh network (WMN) is composed of static mesh routers and mobile mesh clients [1]. Static mesh routers form the infrastructure WMN part, and mobile mesh clients constitute the client WMN part. Unlike static mesh routers, mobile mesh clients have limited energy, and when energy is exhausted, mesh clients cannot be used anymore.

There are two types of data traffic in hybrid WMN. One is gateway-oriented traffic, and the other is client-oriented traffic. In the gateway-oriented traffic, data can be transmitted through gateway to Internet. In the client-oriented traffic, mesh clients will be connected through multiple hops.

Routing protocols can extensively improve the performance of a network, as the performance of the whole network can be completely different depending on each route chosen. Thus, a proper routing protocol design is very essential. Routing protocols can be categorized into proactive, reactive and hybrid routing protocols [2]. Proactive routing protocols suit infrastructure WMN well, and reactive routing protocols adapt to client WMN. Thus, hybrid routing protocols match well to hybrid WMN. However, existing hybrid routing protocols designed for hybrid WMN are quite few. Load condition of neighboring nodes is not sufficiently considered, and different network factors are considered individually. Some undesired performance could be caused. For example, consider the case where a node is with low load itself but in high load region. Although the node itself has low load, its neighbors with high load can transmit many data packets to it. Congestion and bad performance will be caused. Therefore, regional load condition of neighbor nodes should not be overlooked. To overcome the weaknesses of existing hybrid routing protocols, and design a routing method more adaptive to hybrid WMN, a load and energy aware hybrid routing protocol
(LE-HRP) is proposed in this paper. The main contributions of LE-HRP are as follows:

- LE-HRP considers neighboring load condition for both mesh routers and clients. The network performance cannot be improved as a whole if selecting routes only based on the condition of individual nodes. LE-HRP considers the queue length of neighbors and the probability of neighbors transmitting packets to the current node. In this way, the queue length which current node may get from neighbors is available. Besides queue length, bandwidth is also considered to describe the load condition more accurate. Both physical and logical interference are taken into account to calculate bandwidth. As load condition of neighbors is considered, a region with high load can be avoided.

- LE-HRP considers load and energy in both client- and gateway-oriented traffic. For mesh routers, queuing delay is considered to evaluate the load condition. As the energy of mesh clients is limited, in addition to reducing delay, energy is also reserved simultaneously. When the residual energy of mesh clients is low, existing hybrid routing protocols just avoid using these mesh clients. However, these mesh clients sometimes may help the network reduce delay dramatically. In LE-HRP, a mesh client with low energy can still be used only if it can help the network reduce delay significantly. In this way, the route with less delay and more energy can be chosen.

The structure of this paper is: Section II introduces some existing research of routing protocols for hybrid WMN. Section III gives the details of LE-HRP. Section IV shows the simulation results and analyses. The conclusion is in Section V.

\section{EXISTING ROUTING PROTOCOLS FOR HYBRID WMN}

So far, existing routing protocols considering the features of hybrid WMN are few. Most of them are reactive protocols. Some are based on AODV [3]. SafeMesh [4] sets different weight values for mesh routers and clients, and channel utility in data flows is considered. LAM [5] considers interference and solves the link load imbalance problem. NSR [6] considers the stability and gateway selection of WMN. The stability is defined by entropy function according to interference and link utilization. Besides, gateway is selected to balance load. Some are based on DSR [7]. WEED [8] considers the expected endto-end delay, interference and bandwidth. LQSCR [9] considers neighbor stability and link quality based on retransmission counts in MAC layer. There are also some routing protocols designed on the basis of WCETT [10]. D-WCETT [11] changes the fixed parameter in WCETT into dynamic load information, which can avoid network congestion. In addition to the dynamic parameter, 
ELARM [12] also uses energy condition to replace the sum of ETT in WCETT.

Reactive routing protocols cannot take full advantage of static mesh routers. Extra cost and delay can be caused since static mesh routers also find routes once data packets are sent, which is undesired. Hybrid routing protocols can overcome such a weakness, and adapt to the features of hybrid WMN. However, existing hybrid routing protocols are even fewer. HMesh [13] combines OLSR [14] and AODV, and uses them among mesh routers and mesh clients separately. HDV [15] makes use of tree-based routing in infrastructure WMN, and uses AODV in client WMN. Both HMesh and HDV only use the hop count as the routing metric, neglecting network condition. RCA-HRP [16] considers the load of neighboring mesh clients for mesh routers, but neglects the neighboring load for mesh clients. Besides, it only uses queue length to evaluate load, which overlooks the influence of interference and bandwidth. LA-CHRP [17] considers the load condition of current access mesh routers during the access process, but it neglects the regional condition.

\section{LOAD AND ENERGY AWARE HYBRID ROUTING PROTOCOL DESIGN}

LE-HRP considers gateway- and client-oriented traffic respectively. The proactive routing method is used among static mesh routers to serve gateway-oriented traffic, and the reactive routing approach is applied in both mesh routers and mesh clients to serve client-oriented traffic. When the proactive routing table does not have an existing route to destination node, the reactive routing will be used. LE-HRP considers the load condition of neighbor nodes, which can help select a right route to avoid an area with high load. As the improved reactive routing approach is based on AODV, hello packets are broadcasted by each node to maintain neighbor information. The hello packets of LE-HRP also contain the load and queue length information, which will not bring extra cost [18].

The general idea of LE-HRP is to establish reasonable weight values for different mesh nodes, and then set routing metric based on weight values. Regional load condition and different features of different types of mesh nodes are considered. Mesh nodes with less weight values and the path with less routing metric will be selected. Existing hybrid routing protocols only use queue length to measure load condition. However, only considering the queue length is not accurate enough. Even in the case where queue length at the radio interface of a node is quite long, wide bandwidth will still enable to empty queue packets quickly and to result in a short delay. Due to such a reason, the ratio of queue packet size over bandwidth is used in LE-HRP to indicate load condition. This ratio is delay cost by emptying queuing packets. Delay is considered both in gateway- and client-oriented traffic. Moreover, as mesh clients have limited energy, besides delay, energy is also taken into account for them.

\section{A. Expected available bandwidth}

To calculate the time by emptying queue length, the expected bandwidth is an important factor. Interference can influence the bandwidth directly. The interference includes both the physical and logical interference, and LE-HRP considers both types. To evaluate the logical interference, the cross-layer method of channel busy time (CBT) is used [19]. The expression of $C B T$ is

$$
C B T=\frac{\text { TotalTime }- \text { IdleTime }}{\text { TotalTime }}
$$

where TotalTime is the total time of detection, and IdleTime is the time when the channel is not occupied.

The large $C B T$ means the channel has long busy time, which is caused by a lot of interfering nodes contending the channel. Thus, the larger $C B T$, the heavier logical interference.

Then, the available bandwidth of the logical interference at node $i$ can be defined as

$$
B_{\text {logical }}^{i}=B_{\text {total }} \times\left(1-C B T_{i}\right)
$$

where $B_{\text {total }}$ is the nominal bandwidth. The larger $C B T$ will cause larger logical interference and less available bandwidth.

In addition, $I R$ [20] is used to evaluate the physical interference. $I R$ at node $i$ is defined as

$$
I R_{i}=\frac{S I N R_{i}}{S N R_{i}}
$$

where $S I N R_{i}$ and $S N R_{i}$ are signal to interference plus noise ratio and signal to noise ratio respectively. They can be computed according to the signal and interference intensity at node $i$. Therefore the influence of physical and logical interference on available bandwidth at node $i$ can be expressed as

$$
B_{\text {available }}^{i}=B_{\text {logical }}^{i} \times I R_{i}
$$

\section{B. Client-oriented traffic}

Regional load condition is considered in reactive routing for client-oriented traffic. As mesh clients have limited energy, besides delay, energy is also considered. To make full use of mesh routers, weight values of mesh router and client are around 1 and 4 [4]. Thus, in the whole network, the probabilities of mesh routers and mesh clients being used (i.e., $p_{\text {router }}$ and $p_{\text {client }}$ ) are $80 \%$ and $20 \%$ respectively. To show the node-type situation of neighbors of node $i$, a ratio (i.e., $\tau_{i}$ ) is defined as

$$
\tau_{i}=\frac{N_{C}^{i}}{N_{i}}
$$

where $N_{C}^{i}$ is the number of neighbor mesh clients, and $N_{i}$ is the total number of neighbor nodes of node $i$.

Among the same type of nodes with similar weight values, each neighboring node can receive the broadcasted route request message from the current node. All these neighbors have the same ability to forward packets from the current node. Thus, based on the feature of broadcasting and weight values, we assume that a node transmitting packets to one node among the same type of nodes is an event with equal probability. Thus, among mesh routers, the probability of node $q$ transmitting packets to the neighbor mesh router $a$ (i.e., $p_{\mathrm{a}}^{q}$ ) is

$$
p_{a}^{q}=\frac{1}{N_{R}^{q}}
$$

where $N_{R}^{q}$ is the number of neighbor mesh routers of node $q$. Similarly, among mesh clients, the probability of a node $r$ transmitting packets to the neighbor mesh client $j$ (i.e., $p_{j}^{r}$ ) is

$$
p_{j}^{\mathrm{r}}=\frac{1}{N_{C}^{r}}
$$

where $N_{C}^{r}$ is the number of neighbor mesh clients of node $r$.

Thus, conditional probability of node $q$ transmitting packets to the neighbor mesh router $a$ (i.e., $p_{q a}$ ) is

$$
p_{q a}=\left\{\begin{array}{c}
p_{\text {router }} \times p_{a}^{q}=80 \% \times \frac{1}{N_{R}^{q}}, \quad \tau_{q} \neq 0 \\
p_{a}^{q}=\frac{1}{N_{R}^{q}}, \quad \tau_{q}=0
\end{array}\right.
$$




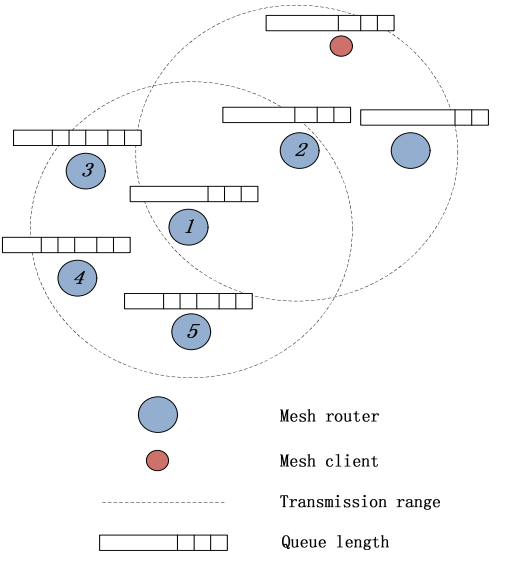

Fig. 1. The example of regional queue length calculation

where $N_{R}^{q}$ is the number of neighbor mesh routers around node $q$. Then the queue length which mesh router $a$ may get from its neighbor nodes (i.e., $Q_{a}^{g e t}$ ) is

$$
Q_{a}^{g e t}=\sum_{q \in M_{a}}\left(Q_{q} \times p_{q a}\right)
$$

where $M_{a}$ is the neighbor set of mesh router $a . Q_{q}$ is the queue length of neighbor node $q$.

To show the calculation of regional queue length clearly, an example is given in Fig. 1. Mesh router 2 has two neighbor mesh routers and one neighbor mesh client. According to formula (8), the probability of mesh router 2 transporting packets to mesh router 1 is 0.4 . If the queue length of mesh router 2 is 5 , then the queue length which mesh router 1 may get from mesh router 2 is 2 . Similarly, the queue lengths that mesh router 1 can also get from its neighbor mesh router 3, 4, 5 can be obtained. Then $Q_{1}^{\text {get }}$ can be obtained according to formula (9).

Then the weight value of mesh router $a$ (i.e., $W_{a}$ ) is set as

$$
W_{a}=D_{a}=\frac{\left(Q_{a}^{g e t}+Q_{a}\right) \times \text { PacketSize }}{B_{\text {available }}^{a}}
$$

where $Q_{a}$ is the queue length currently at mesh router $a$. $W_{a}$ means the delay cost by emptying the data packets at node $a$.

For a mesh client $j$, the conditional probability of node $r$ transmitting packets to the neighbor mesh client $j$ (i.e., $p_{r j}$ ) is

$$
p_{r j}=\left\{\begin{array}{c}
p_{\text {client }} \times p_{j}^{r}=20 \% \times \frac{1}{N_{C}^{r}}, \quad \tau_{r} \neq 1 \\
p_{j}^{r}=\frac{1}{N_{C}^{r}}, \quad \tau_{r}=1
\end{array}\right.
$$

where $N_{C}^{r}$ is the number of neighbor mesh routers around node $r$. Then similar to the mesh router $a$, the queue length mesh client $j$ may get from its neighbors (i.e., $Q_{j}^{\text {get }}$ ), and the delay of emptying packets at mesh client $j$ (i.e., $D_{j}$ ) can be obtained.

Besides delay and regional load, energy is another important factor for mesh clients. All existing hybrid routing protocols consider energy and other performance metrics separately. When a mesh client has less energy, it will be just avoided being used when finding routes. LE-HRP considers delay and energy at the same time.

The total number of packets that mesh client $j$ can transmit (i.e., $P k t N u m_{j}$ ) is

$$
\operatorname{PktNum}_{j}=\frac{E_{\text {left }}^{j}}{E_{r}+E_{t}}
$$

where $E_{\text {left }}^{j}$ is the residual energy of mesh client $j . E_{r}$ and $E_{t}$ are energy cost by receiving and transferring a packet respectively. Then the time cost by emptying all these packets at mesh client $j$ (i.e., $T_{\text {life }}^{j}$ ) is

$$
T_{\text {life }}^{j}=\frac{\text { PktNum }_{j} \times \text { PacketSize }}{B_{\text {available }}^{j}}
$$

According to $T_{l i f e}^{j}$ and $D_{j}$, the batch number which mesh client $j$ can still be used (i.e., $n_{j}$ ) is

$$
n_{j}=\frac{T_{\text {life }}^{j}}{D_{j}}=\frac{\frac{P k t N u m_{j} \times \text { PacketSize }}{B_{\text {available }}}}{\frac{\left(Q_{j}^{\text {get }}+Q_{j}\right) \times \text { PacketSize }}{B_{\text {available }}^{j}}}=\frac{P k t N u m_{j}}{\left(Q_{j}^{\text {get }}+Q_{j}\right)}
$$

where $Q_{j}$ is the current queue length at mesh client $j$.

So $n_{j}$ is the total times that mesh client $j$ can still be used to forward packets. Not only the residual energy and lifetime is considered like existing hybrid routing protocols, the time needed to process queue is also taken into account. The client with a little more energy but very long queue will not be selected, which is more reasonable. To make the weight value of mesh client $j$ around 4 [4], the weight (i.e., $W_{j}$ ) is set as

$$
W_{j}=\frac{1}{n_{j}}+4=\frac{D_{j}}{T_{\text {life }}^{j}}+4=\frac{\left(Q_{j}^{\text {get }}+Q_{j}\right)}{\text { PktNum }}+4
$$

The less $W_{j}$ means the more batch number that mesh client $j$ can be used. Energy and delay are considered in $W_{j}$ simultaneously. For example, if mesh client $j$ has the residual energy which is only half the other mesh client, but it can obtain less than half of delay, mesh client $j$ will be chosen. That is, a mesh client with lower energy can be used if it can help the network reduce the delay more significantly. On the other hand, if the delay of different mesh clients are similar, a mesh client with more residual energy will be chosen. In this way, delay and energy is improved as a whole. To meet the fairness QoS constraint, when the residual energy of mesh clients is lower than $10 \%$, this means that the nodes are really low on the battery level and should only be used if there is no other cost-effective alternative. To avoid using this mesh client as far as possible, $W_{j}$ is set to be 10 [17] to fully distinguish it from others.

On the basis of weight values of mesh router and mesh client, the routing metric of reactive routing (i.e., $M e t r i c_{r e a}^{h}$ ) is

$$
\text { Metric }_{\text {rea }}^{h}=\sum_{\text {router } a \in h} W_{a}+\sum_{\text {client } j \in h} W_{j}
$$

where $h$ is the reactive path where mesh router $a$ and mesh client $j$ locate. According to this node type aware routing metric, a route with more static mesh routers will be selected. In this way, energy and delay can be improved simultaneously.

\section{Gateway-oriented traffic}

In LE-HRP, the proactive routing method is used among infrastructure WMN to serve gateway-oriented traffic. The regional load condition is also considered, which is similar to that in reactive routing method mentioned above.

The way of setting weight values for mesh routers in the client-oriented traffic is also used here. As proactive routes are 
TABLE I

SIMULATION PARAMETERS

\begin{tabular}{ll}
\hline Simulation Parameters & Values \\
\hline Simulation time & $100 \mathrm{~s}$ \\
Traffic type & UDP \\
Packet size & 1024 bytes \\
Packet rate & $80 \mathrm{kbps}$ \\
Initial energy of each router & $10000 \mathrm{~J}$ \\
Initial energy of each client & $500 \mathrm{~J}$ \\
Transmission range & $250 \mathrm{~m}$ \\
Interference range & $550 \mathrm{~m}$ \\
Propagation model & Two Ray Ground \\
Mobility model & Random direction 2d mobility model \\
Antenna & Omnidirectional \\
\hline
\end{tabular}

composed of static mesh routers, the routing metric of proactive routing method (i.e., Metric $c_{\text {pro }}^{p}$ ) is

$$
\text { Metric pro }{ }_{\text {pro }}^{p} \sum_{i \in p} W_{i}=\sum_{i \in p} \frac{\left(Q_{i}^{\text {get }}+Q_{i}\right) \times \text { PacketSize }}{B_{\text {available }}^{i}}
$$

where $p$ is the proactive path where mesh router $i$ locates. So the proactive path with less delay will be selected.

\section{Simulation eValuation}

\section{A. Simulation environment}

The simulation is implemented in an area of $1000 \mathrm{~m} \times 1000 \mathrm{~m}$ through NS3 [21]. 25 static mesh routers are deployed into a grid, and 50 mobile mesh clients move randomly. As hello packets contain the queue length information, mesh nodes can maintain neighbors' information and compute weights easily. AODV is compared as a fully reactive routing protocol. Hybrid routing protocols HMesh and LA-CHRP are also compared with LEHRP. Constant bit rate data stream is sent, and the sources and destinations are chosen randomly. Detailed simulation parameters are shown in Table I.

\section{B. Performance metrics}

- Average packet loss rate: the ratio of lost packet number to the number of total packets sent by source node.

- Average delay: the time period cost by delivering a packet from source to destination successfully.

- Average network throughput: an end-to-end metric to show the number of bit which can be transmitted successfully in unit time.

- Average energy consumption of mesh clients: the consumed energy of mesh clients to deliver each packet in success.

- The minimum residual energy of mesh clients: the minimum remaining energy of mesh clients in the whole network at the end of the simulation.

\section{Simulation results and analyses}

With different number of flows and speed of mesh clients, the simulation results in terms of different performance metrics are shown in Fig. 2-Fig. 6. When the number of flows is varied, the speed of mesh clients is $2 \mathrm{~m} / \mathrm{s}$ and when the clients' speed is varied the number of flows is 8 . For the reason that mesh clients move randomly, the topology of network is not fixed and the relationship between mesh nodes is changing. Therefore, the simulation results may fluctuate irregularly.

From Fig. 2, LE-HRP has lower average packet loss rate. Unlike LA-CHRP only considering the load of current node,

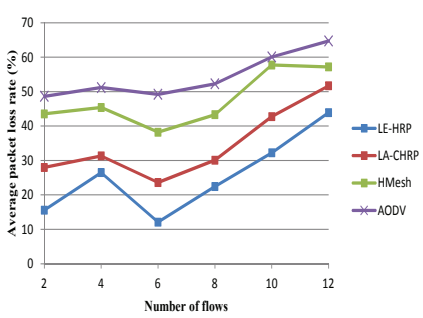

(a) Different number of flows

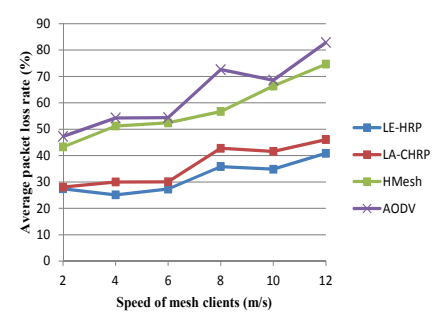

(b) Different speed of mesh clients
Fig. 2. Average packet loss rate

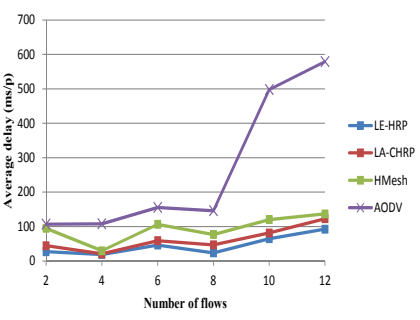

(a) Different number of flows

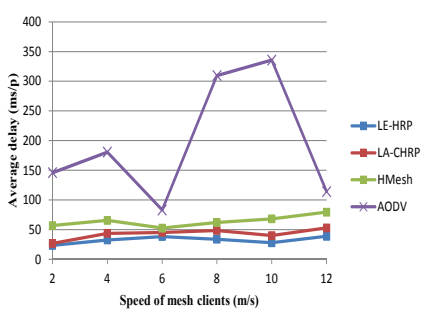

(b) Different speed of mesh clients
Fig. 3. Average delay

LE-HRP considers load of neighboring nodes, and the area with high load can be avoided during route discovery. In addition, LEHRP gives priority to mesh routers when selects routes. The path will be more stable than HMesh, and mobile mesh clients have less influence on LE-HRP. As a result, LE-HRP leads to a better regional condition and obtains less average packet loss rate when the number of flows and mesh clients' speed are changing.

Fig. 3 shows LE-HRP has less average delay. Delay is considered in both gateway- and client-oriented traffic in LE-HRP. Thus, LE-HRP can find routes with less delay. AODV has most delay because it is a fully reactive routing protocol, and all mesh nodes in the network have to discover routes every time. No routing tables are stored before packet transmission.

In Fig. 4, LE-HRP has higher average network throughput. The network throughput does not linearly increase against the number of flows because of the variable topology, changeable interference relationship and randomly selected sources and destinations. When calculate bandwidth, LE-HRP considers both physical and logical interference, so it can select routes with less interference and wider bandwidth than LA-CHRP and HMesh. Besides, as the regional condition is taken into account, the network performance can be improved as a whole. LE-HRP can also select mesh routers in priority, which brings more stable route. When the mesh clients' speed is changing, the network throughput will not decrease dramatically like HMesh. As a result, LE-HRP can obtain high network throughput.

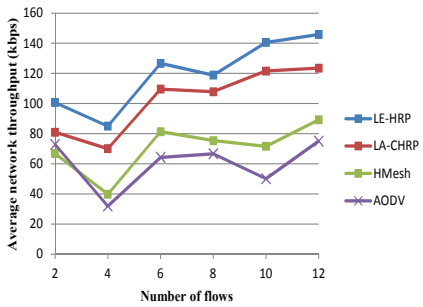

(a) Different number of flows

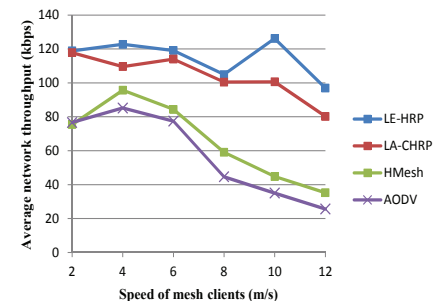

(b) Different speed of mesh clients
Fig. 4. Average network throughput 


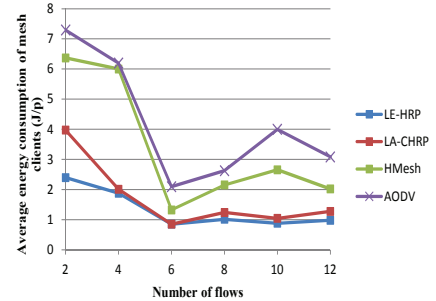

(a) Different number of flows

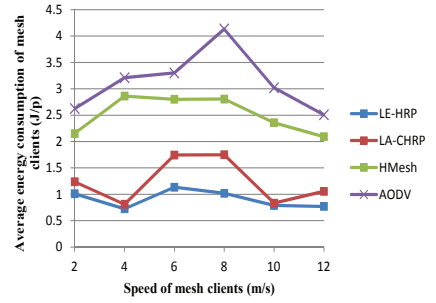

(b) Different speed of mesh clients
Fig. 5. Average energy consumption of mesh clients

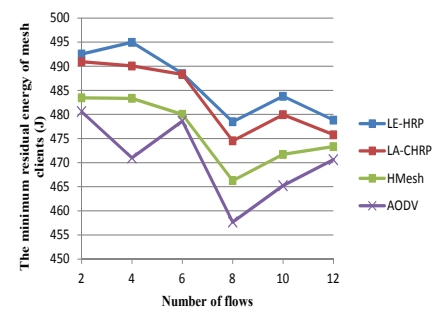

(a) Different number of flows

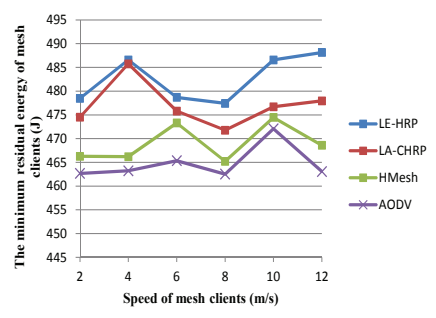

(b) Different speed of mesh clients
Fig. 6. The minimum residual energy of mesh clients

From Fig. 5, it can be seen that LE-HRP has less average energy consumption of mesh clients. As mesh clients have limited energy, besides delay, LE-HRP also improves energy. Due to the node type aware routing metric, mesh routers will be used in priority, which can avoid using mesh clients too much. In addition, the mesh clients with heavy regional load is also avoided. The amount of packets needed to be forwarded by mesh clients is further reduced. Then, the energy consumption of mesh clients is less than LA-CHRP and HMesh.

LE-HRP has more minimum residual energy of mesh clients in Fig. 6. LE-HRP can use energy more effectively with balance. The mesh client with more energy has higher probability to be used. The mesh client with low energy can be used only if it can help the network reduce the delay significantly. In this way, LE-HRP can effectively balance the delay reduction and energy consumption. Due to the movement, if the neighbors of source nodes are all mesh clients, sources have to transmit packets to mesh clients, and the energy consumption will be high. Therefore, the energy consumption and residual energy are related to the node location and network topology. As the network topology is always changing, the residual energy is not strictly linearly decrease against the number of flows.

\section{CONCLUSION}

Hybrid routing protocol including both proactive and reactive routing methods is very effective for hybrid WMN. Existing hybrid routing protocols are few with limitations. Different network factors are considered separately. LE-HRP considers the regional load condition. Reducing delay and enhancing energy efficiency are considered simultaneously for mesh clients. Besides, LE-HRP considers both gateway- and client-oriented traffic. In proactive routing metric for gateway-oriented traffic, delay is taken into account. To calculate delay, the queue length which can be obtained from neighbor nodes and bandwidth at current node are considered. Both physical and logical interference is taken into account. In the node type aware reactive routing metric for client-oriented traffic, besides delay, energy is also considered for mesh clients. LE-HRP enables a mesh client with low energy to be used if it can help the network reduce delay significantly. Otherwise, the mesh client with more energy will be selected. Simulation results through NS3 show that LE-HRP can get better network performance.

\section{REFERENCES}

[1] Y. Liu, K. F. Tong, X. Qiu, Y. Liu, and X. Ding, "Wireless mesh networks in IoT networks," in IEEE International Workshop on Electromagnetics: Applications and Student Innovation Competition, pp. 183-185, London, UK, May 2017.

[2] S. D. Samo, and J. L. E. Fendji, "Evaluation of Energy Consumption of Proactive, Reactive, and Hybrid Routing Protocols in Wireless Mesh Networks Using 802.11 Standards," Journal of Computer and Communications, vol. 6, no. 4, pp. 1-30, Apr. 2018.

[3] C. Perkins, E. Belding-Royer, and S. Das, "Ad hoc on-demand distance vector (AODV) routing," No. RFC 3561, 2003.

[4] A. A. Pirzada, M. Portmann, R. Wishart, and J. Indulska, "SafeMesh: A wireless mesh network routing protocol for incident area communications," Pervasive and Mobile Computing, vol. 5, no. 2, pp. 201-221, Apr. 2009.

[5] Z. Hao, and Y. Li, "An adaptive load-aware routing algorithm for multiinterface wireless mesh networks," Wireless Networks, vol. 21, no. 2, pp. 557-564, Feb. 2015.

[6] M. Boushaba, A. Hafid, and M. Gendreau, "Node stability-based routing in wireless mesh networks," Journal of Network and Computer Applications, vol. 93, pp. 1-12, Sep. 2017.

[7] D. B. Johnson, D. A. Maltz, and J. Broch, "DSR: The dynamic source routing protocol for multi-hop wireless ad hoc networks," Ad Hoc Networking, vol. 5, pp. 139-172, Jan. 2001.

[8] H. Li, Y. Cheng, C. Zhou, and W. Zhuang, "Minimizing end-to-end delay: A novel routing metric for multi-radio wireless mesh networks," in IEEE INFOCOM, pp. 46-54, Rio de Janeiro, Brazil, Apr. 2009.

[9] W. Song, and X. Fang, "Cross-layer routing with link quality and stabilityaware in ITS hybrid wireless mesh networks," in IEEE 6th International Conference on Advanced Language Processing and Web Information Technology, pp. 304-308, Henan, China, Aug. 2007.

[10] R. Draves, J. Padhye, and B. Zill, "Routing in multi-radio, multi-hop wireless mesh networks," in Proc. of the 10th Annual International Conference on Mobile Computing and Networking, pp. 114-128, Philadelphia, USA, Sep. 2004.

[11] R. F. Ali, A. K. Kiani, and A. A. Pirzada, "Load dependent dynamic path selection in multi-radio hybrid wireless mesh networks," in Wireless Communications and Networking Conference (WCNC), pp. 2020-2025, Istanbul, Turkey, Apr. 2014.

[12] A. K. Kiani, R. F. Ali, and U. Rashid, "Energy-load aware routing metric for hybrid wireless mesh networks," in Vehicular Technology Conference (VTC Spring),pp. 1-5, Glasgow, UK, May 2015.

[13] L. Zhao, Z. Yu, J. Niu, H. Zhang, and W. Ding, "A hybrid routing protocol for hierarchy wireless mesh networks," in 6th International Conference on Wireless Communications Networking and Mobile Computing (WiCOM), pp. 1-4, Chengdu, China, Sept. 2010.

[14] T. Clausen, and P. Jacquet, "Optimized link state routing protocol (OLSR)," No. RFC 3626, 2003.

[15] A. N. Le, D. W. Kum, and Y. Z. Cho, "An efficient hybrid routing approach for hybrid wireless mesh networks," Advances in Information Security and Assurance, vol. 5576, pp. 532-542, Jun. 2009.

[16] Y. Chai, and X. J. Zeng, "Regional condition-aware hybrid routing protocol for hybrid wireless mesh network," Computer Networks, vol. 148, pp. 120128, Jan. 2019.

[17] Y. Chai, W. Shi, and T. Shi, "Load-aware cooperative hybrid routing protocol in hybrid wireless mesh networks," AEU-International Journal of Electronics and Communications, vol. 74, pp. 135-144, Apr. 2017.

[18] P. Kyasanur, and N.H. Vaidya, "Routing and link-layer protocols for multichannel multi-interface ad hoc wireless networks," in ACM SIGMOBILE Mobile Computing and Communications Review, pp. 31-43, New York, USA, Jan. 2006.

[19] V. C. Borges, M. Curado, and E. Monteiro, "Cross-layer routing metrics for mesh networks: Current status and research directions," Computer Communications, vol. 34, no. 6, pp. 681-703, May 2011.

[20] V. C. Borges, D. Vieira, M. Curado, and E. Monteiro, "Routing metric for interference and channel diversity in multi-radio wireless mesh network," in 8th International Conference on Ad-Hoc Networks and Wireless, pp. 55-68, Berlin, Heidelberg, Sept. 2009.

[21] ns-3. http://www.nsnam.org/. Accessed January 9, 2018. 